\title{
Probing the Transition from Hydrophilic to Hydrophobic Solvation with Atomic Scale Resolution
}

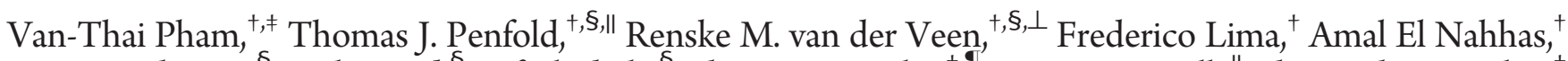 \\ Steve L. Johnson, ${ }^{\S}$ Paul Beaud, ${ }^{\S}$ Rafael Abela, ${ }^{\S}$ Christian Bressler, ${ }^{+}{ }^{\uparrow}$ Ivano Tavernelli," Christopher J. Milne, ${ }^{+}$ \\ and Majed Chergui ${ }^{*,+}$ \\ ${ }^{\dagger}$ Ecole Polytechnique Fédérale de Lausanne, Laboratoire de Spectroscopie Ultrarapide, ISIC-FSB, Station 6, CH-1015 Lausanne,
Switzerland
${ }^{\S}$ Swiss Light Source, Paul-Scherrer-Institut, CH-5232 PSI-Villigen, Switzerland
${ }^{\|}$Ecole Polytechnique Fédérale de Lausanne, Laboratoire de Chimie Et Biochimie Computationnelles, ISIC-FSB,
CH-1015 Lausanne, Switzerland
}

Supporting Information

ABSTRACT: Picosecond and femtosecond X-ray absorption spectroscopy is used to probe the changes of the solvent shell structure upon electron abstraction of aqueous iodide using an ultrashort laser pulse. The transient $\mathrm{L}_{1,3}$ edge EXAFS at 50 ps time delay points to the formation of an expanded water cavity around the iodine atom, in good agreement with classical and quantum mechanical/molecular mechanics (QM/MM) molecular dynamics (MD) simulations. These also show that while the hydrogen atoms pointed toward iodide, they predominantly point toward the bulk solvent in the case of iodine, suggesting a hydrophobic behavior. This is further confirmed by quantum chemical (QC) calculations of $\mathrm{I}^{-} / \mathrm{I}^{0}\left(\mathrm{H}_{2} \mathrm{O}\right)_{n=1-4}$ clusters. The $\mathrm{L}_{1}$ edge sub-picosecond

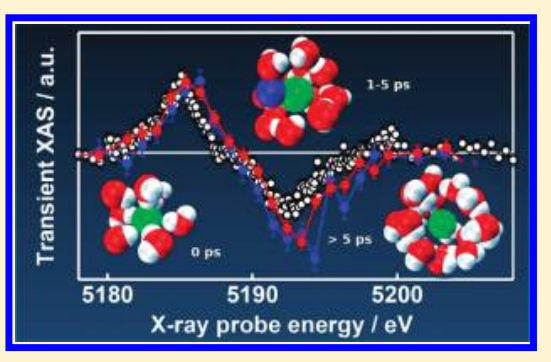
spectra point to the existence of a transient species that is not present at $50 \mathrm{ps}$. The QC calculations and the QM/MM MD simulations identify this transient species as an $\mathrm{I}^{0}\left(\mathrm{OH}_{2}\right)$ complex inside the cavity. The simulations show that upon electron abstraction most of the water molecules move away from iodine, while one comes closer to form the complex that lives for 3-4 ps. This time is governed by the reorganization of the main solvation shell, basically the time it takes for the water molecules to reform an H-bond network. Only then is the interaction with the solvation shell strong enough to pull the water molecule of the complex toward the bulk solvent. Overall, much of the behavior at early times is determined by the reorientational dynamics of water molecules and the formation of a complete network of hydrogen bonded molecules in the first solvation shell.

\section{INTRODUCTION}

Hydrophobicity is considered the major driving force behind fundamental biological processes. ${ }^{1,2}$ It also plays a role in materials science such as in the case of water in zeolites, fluid flow in small channels (e.g., carbon nanotubes), or evaporation and hydration of nanoparticles. ${ }^{3}$ It is now generally recognized that hydrophobicity manifests itself differently on small and large length scales. ${ }^{4}$ For example, in the hydration of small hydrophobic species (which are of interest here), these can fit into the water hydrogen-bond network without destroying any hydrogen bonds, ${ }^{5}$ implying that the enthalpy of solvation is small. The formation of small cavities in the solvent to accommodate the solute is an entropically dominated process, and the presence of the solute constrains the orientational and translational degrees of freedom of the neighboring water molecules. The microscopic understanding of hydrophobic hydration relies so far almost solely on theory ${ }^{6,7}$ and simulations. ${ }^{4,8-10}$ This is due to the fact that experimentally probing hydrophobicity at the atomic scale is very difficult, and quantitative studies are not yet available. For solid-water interfaces, these have mostly been high-energy $\mathrm{X}$-ray reflectivity measurements. ${ }^{11,12}$ In the case of atomic species in solutions, Neilson and co-workers ${ }^{13,14}$ used the technique of isotopic substitution neutron diffraction and were able to extract structural information related to the $\mathrm{Ar}-\mathrm{O}$ and $\mathrm{Ar}-\mathrm{D} / \mathrm{H}$ partial radial distribution functions for the $\mathrm{Ar}-\mathrm{D}_{2} \mathrm{O} / \mathrm{H}_{2} \mathrm{O}$ system, in good agreement with computer simulations. ${ }^{15}$ Bowron, Filipponi and co-workers used X-ray absorption spectroscopy (XAS) for determining the solvent shell structure around $\mathrm{Kr}$ atoms dissolved in water under pressure, ${ }^{16-18}$ via the analysis of the X-ray absorption near edge structure (XANES) and extended X-ray absorption fine structure (EXAFS). They extracted solute-oxygen radial distribution functions pointing to an ordering of the water oxygen correlations around the $\mathrm{Kr}$ atom through the existence of a well-defined hydration structure.

Very few time-resolved studies of hydrophobicity have been reported so far. Rezus and Bakker ${ }^{19}$ carried out a femtosecond mid-infrared spectroscopy study of the orientational mobility of water molecules in the hydration shells of hydrophobic groups. They concluded that hydrophobic groups are surrounded by a

Received: $\quad$ May 11, 2011

Published: July 08, 2011 
number of water molecules that display much slower orientational dynamics than the bulk liquid and are therefore effectively immobilized. They found that typically each methyl group is surrounded by four immobilized water $\mathrm{OH}$ groups, thus, supporting from a dynamical perspective the so-called iceberg picture $^{20}$ that was put forward to explain hydrophobicity. In this picture, the water molecules around hydrophobic groups form rigid, ice-like structures, and the hydrophobic effect originates from the freeing of entropy associated with the transfer of water molecules from the solvation shell to the bulk. However, this picture is in conflict with NMR and dielectric relaxation studies, ${ }^{21-24}$ and simulations studies. ${ }^{25,26}$ Very recently, Laage et al. ${ }^{27}$ through molecular dynamics simulations and an analytic jump reorientation model, also found no evidence for immobilized water molecules around hydrophobic solutes. Their moderate rotational slowdown compared to bulk water is mainly due to slower hydrogen-bond exchange. The slowdown is quantitatively described by a solute excluded volume effect at the transition state for the key hydrogen-bond exchange in the reorientation mechanism. This picture is consistent with both the mid-IR ultrafast spectroscopy and the NMR results and yields quantitative predictions of the rotational slowdown for diverse hydrophobic solutes of varying size over a wide concentration range. The authors could also explain why hydrophobic groups slow water reorientation less than do some hydrophilic groups.

Indeed, of importance for chemistry in aqueous media is the contrast between hydrophobic and hydrophilic behavior. One of the features that distinguishes hydrophilic from hydrophobic behavior is the shift in the mobilities of the anions with respect to the cations as a function of size, which can be traced to the different orientation of water in the primary solvation shell of the ions $(\mathrm{H}$ atoms pointing toward anions and oxygen atoms toward cations). ${ }^{8,9,28,29}$ Using a simple point charge model for water and charged spherical Lennard-Jones solutes, along with an extended Lagrangian or Hamiltonian in which the charge and the size of the ions are considered as dynamical variables, Lynden-Bell, Rasaiah and co-workers calculated entropies of solvation of halogen atoms as a function of the charge, introducing the terms hydrophobic and hydrophilic solvation. 8 "They showed that the entropy of solvation of a solute passes through a minimum near zero charge, signaling cavity formation (hydrophobic hydration) with the water molecules held together by $\mathrm{H}$-bonds. The solvation entropy increases gradually as the solute is charged positively or negatively, reflecting break-up of the cavity. Indeed, their simulations showed that the solvation shell radius increases from $3.6 \AA$ for $\mathrm{I}^{-}$to $4.25 \AA$ for $\mathrm{I}^{0}$ with the hydration numbers (i.e., the number of molecules in the first solvation shell) going from 7.9 for $\mathrm{I}^{-}$to 27 for $\mathrm{I}^{0}$.

The contrast between the solvation shell structures of aqueous iodide and iodine, which are the systems of interest here, can be investigated by laser abstraction of the electron from aqueous iodide and probing of the formed iodine atom by time-resolved XAS. $^{30-34}$ We previously presented ${ }^{35}$ a preliminary picosecond XAS study of multiphoton-excited aqueous iodide, which showed significant changes in the XANES and EXAFS regions of the $\mathrm{L}_{1,3}$ edges $50 \mathrm{ps}$ after excitation, suggesting an extensive solvent rearrangement in the transition from iodide to iodine. However, these changes were not quantified by lack of a precise determination of the photolysis yield, which is a crucial parameter for the structural analysis. ${ }^{32}$ Soon after, Elles et al. ${ }^{36}$ reported a similar study on aqueous bromide excited into the so-called charge-transfer-to-solvent (CTTS) bands by one-photon at $200 \mathrm{~nm}$, and probing of the resulting neutral bromine atom was done at its K-edge using picosecond hard X-ray pulses. Their recovered $\mathrm{Br}^{0} \mathrm{~K}$-edge spectrum showed shallow features above the edge, making it difficult to extract a solvent shell structure. For this purpose, they carried out Monte Carlo simulations of the radial distribution functions ( $\mathrm{RDF}$ ) of $\mathrm{Br}^{-}$and $\mathrm{Br}^{\circ}$, and found that indeed in the latter case, the solvent shell structure is more diffuse and expands by $\sim 0.5 \AA$, explaining this way the weak modulations of the spectrum above the $\mathrm{Br}^{\circ} \mathrm{K}$-edge. Their RDF also exhibited a small peak at shorter distances, which they attributed to formation of a three-electron $\left(\sigma^{2} \sigma^{1 *}\right)$ bonded $\mathrm{Br}^{0} \cdots \mathrm{OH}_{2}$ complex, although no signature of this complex was found in their time-resolved $\mathrm{X}$-ray signal.

There is a vast literature of ultrafast optical studies of photoexcited aqueous iodide that have investigated the formation of the aqueous electron via the CTTS bands in the UV below $260 \mathrm{~nm},{ }^{37-39}$ or by multiphoton excitation. ${ }^{40,41}$ The electron was detected by its broad and intense absorption centered around $800 \mathrm{~nm}$, while in one case only was the iodine atom probed in the picosecond regime by its weak broad absorption centered at $260 \mathrm{~nm}^{38}$ This band overlaps the intense iodide CTTS bands and the blue tail of the solvated electron absorption band, making the direct detection of neutral atomic iodine rather ambiguous. Furthermore, optical domain spectroscopy cannot deliver information about the structure of the solvent shell.

In a recent paper, ${ }^{42}$ we showed that the $\mathrm{L}_{3}$-edge EXAFS of aqueous iodide is best reproduced by simulated spectra derived from the $\mathrm{I}^{-}-\mathrm{O}$ and $\mathrm{I}^{-}-\mathrm{H}$ RDFs obtained from quantum mechanics/molecular mechanics $(\mathrm{QM} / \mathrm{MM})^{43}$ molecular dynamics (MD) simulations. These simulations showed that the water molecules of the first solvation shell have at least one of their $\mathrm{H}$-atoms pointing toward the iodide. The RDFs yielded an average $\mathrm{I}-\mathrm{H}$ distance of $\sim 2.6 \AA$ and an average $\mathrm{I}-\mathrm{O}$ distance of $\sim 3.5 \AA$. We also identified a weak anisotropy of the solvent shell, which had already been hinted to by Wick et al. ${ }^{44}$ Here, we report on picosecond and femtosecond studies of the $\mathrm{L}_{1,3}$ XANES and EXAFS upon multi photon excitation of aqueous iodide at $400 \mathrm{~nm}$ to probe the evolution of the solvation shell structure from the iodide (hydrophilic) to the iodine case. Compared to the above-mentioned ultrafast optical studies, X-ray absorption spectroscopy offers the advantage of interrogating the electronic structure of the solute (rather than the electron) as well as the geometric structure of its environment (i.e., the solvation shell). From the picosecond $\mathrm{L}_{3}$-edge transient EXAFS, we determine the solvent structure around $\mathrm{I}^{0}$ at $50 \mathrm{ps}$, by comparing with the transients calculated from classical (nonpolarizable) and QM/ MM MD simulations of iodine and the QM/MM MD simulations of iodide. ${ }^{42}$ Our data show the formation of an expanded cage around $\mathrm{I}^{0}$ which we attribute to hydrophobic solvation based on $\mathrm{MD}$ simulations and quantum chemical (QC) calculations of $\mathrm{I}^{0}\left(\mathrm{H}_{2} \mathrm{O}\right)_{n=1-4}$ clusters. Furthermore, QM/MM MD simulations of an $\mathrm{I}^{\mathrm{O}}\left(\mathrm{H}_{2} \mathrm{O}\right)_{9}$ cluster in bulk classical water point to the formation of a weakly bound $\mathrm{I}^{0}\left(\mathrm{OH}_{2}\right)$ complex, which is short-lived, typically a few picoseconds, before the water molecule of the complex merges back into the bulk. The lifetime of the complex is determined by the time it takes to complete the formation of a cavity of hydrogen bonded molecules around the solute (hydrophobic solvation) following electron abstraction from iodide. The formation of the complex gives rise to a shift of the $2 s-5 p$ resonance at the $L_{1}$-edge, which we observe by femtosecond XAS. 


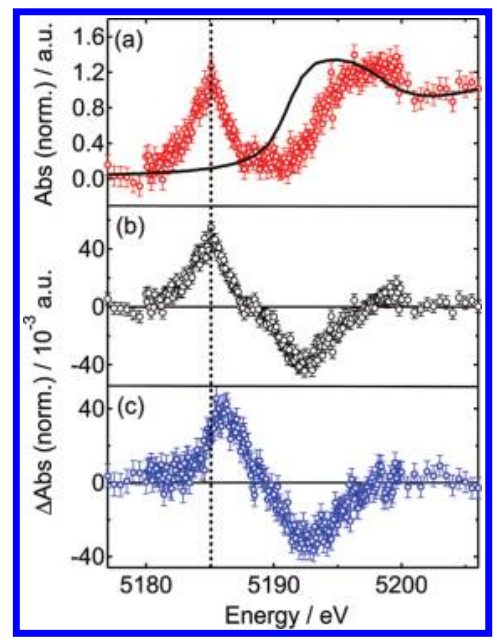

Figure 1. (a) Normalized static iodide X-ray absorption spectrum at the $\mathrm{L}_{1}$ edge (solid line) and reconstructed $\mathrm{I}^{0}$ spectrum using the transient spectrum at 50 ps time delay (b) and the photolysis yield of $4.8 \%$ (see Supporting Information SIV.2) for a $50 \mathrm{mM}$ sample concentration; (b) normalized transient spectra (difference between laser excited minus laser unexcited X-ray absorption spectra) at a time delays of $50 \mathrm{ps}$; (c) same as panel $b$, but at a time delay of $2 \mathrm{~ns}$.

The experimental procedure for the static and the picosecond and femtosecond XAS experiments was already described in refs 32, 45 and 46 and is presented in detail in the Supporting Information (SI). Briefly, the sample consists of a flowing liquid jet of aqueous iodide which is excited at a repetition rate of $1 \mathrm{kHz}$ by $400 \mathrm{~nm}$ femtosecond laser pulses. The sample is probed in absorption by $\sim 60$ ps hard X-ray pulses from the synchrotron. For the sub-picosecond experiments, femtosecond X-ray pulses are generated using the so-called slicing scheme (see Supporting Information SI.3). In both cases the X-ray absorption through the sample is sampled at $2 \mathrm{kHz}$, and the transient (difference between excited minus unexcited sample absorption) spectrum is recorded on line. The use of $400 \mathrm{~nm}$ excitation, which is well below the CTTS states of iodide $(<260 \mathrm{~nm})$, implies a multi photon excitation process for the electron abstraction. Indeed, the dependence of solvated electron absorption as a function of pump laser power is found to be between quadratic and cubic (Figure S1). Because the CTTS band lying at $200 \mathrm{~nm}$ is a strong one-photon allowed transition, it is likely to be weakly twophoton absorbing. We conclude that the excitation is therefore predominantly three-photon, which ensures that the iodide is directly excited well into the ionization continuum, while the electron is ejected far from the excited center. ${ }^{39}$ Details of the procedures for the $\mathrm{MD}$ simulations and for the $\mathrm{QC}$ calculations are also presented in the Supporting Information.

\section{RESULTS}

2.1. Picosecond Transients. 2.1.1. $L_{1}$-Edge Spectra. Figure 1 shows the static iodide $\mathrm{L}_{1}$ XANES spectrum (solid line) along with the transient (laser excited minus unexcited X-ray absorption spectrum of the sample) obtained at $50 \mathrm{ps}$ (b) and $2 \mathrm{~ns}$ (c) time delays for a sample of $50 \mathrm{mM}$ concentration. They show positive and negative features near 5185 and $5192 \mathrm{eV}$, respectively. The positive peak is due to the bound-bound $2 s-5 p$ transition, which appears after the electron abstraction leaves a hole in the $5 \mathrm{p}$ orbital. ${ }^{35}$ This feature is therefore a fingerprint of

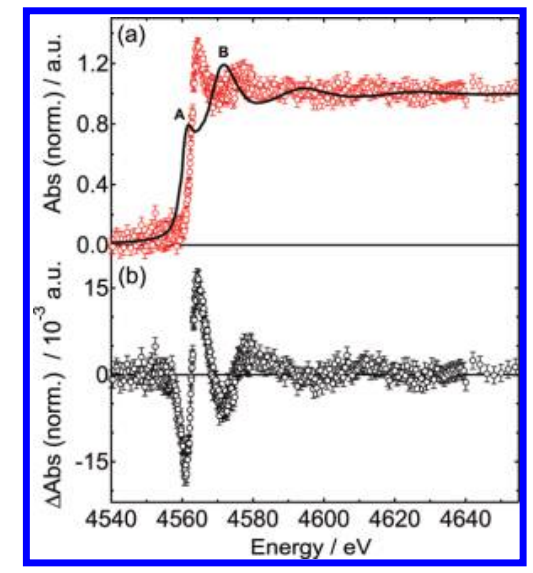

Figure 2. Normalized static iodide $\mathrm{L}_{3}$ edge XAS (solid line) and reconstructed $\mathrm{I}^{0}$ spectrum using the transient spectrum at $50 \mathrm{ps}$ time delay (b) and the photolysis yield of 3\% (see Supporting Information SIV.3), sample concentration $100 \mathrm{mM}$; (b) normalized $\mathrm{L}_{3}$ edge transient spectrum at a time delays of $50 \mathrm{ps}$.

neutral iodine at early times, as we demonstrate in the Supporting Information that at $50 \mathrm{ps}$ time delay, the sample contains only $\mathrm{I}^{\mathrm{D}}$ and the solvated electron as photoproducts. The negative feature is due to the blue shift of the absorption edge when the oxidation state of the solute changes from -1 to 0 . In Figure 1c, the positive feature shifts to higher energies and is broader on its high energy side. This is due to formation of subsequent products, predominantly $\mathrm{I}_{2}^{-}$, as will be discussed later. The time evolution of the positive signal was recorded up to several nanoseconds (not shown here) and it reflects the kinetics of electron-iodine recombination (eq. S6) and diidode formation (eq. S8) contained in the rate eqs $\mathrm{S} 17$.

2.1.2. $\mathrm{L}_{3}$-Edge Spectra. Figure 2 shows the static $\mathrm{L}_{3}$ edge spectrum of iodide (solid line, a) and the transient spectrum recorded at $50 \mathrm{ps}$ delay (b) for a sample of $100 \mathrm{mM}$ concentration. The former exhibits features, labeled A and B, which were shown to be multiple scattering (MS) XANES features ${ }^{47}$ and therefore bear information about the 3-D structure of the solvent shell. Clear photo induced changes are seen in the region of these features, but also at higher energies in the EXAFS region. The changes above the edge are more pronounced than those at the $L_{1}$ edge. Therefore, we will later concentrate on the $L_{3}$ edge for the structural analysis of the solvent shell. The transient exhibits a first negative feature at $4560.8 \mathrm{eV}$, which we later assign to the oxidation (blue) shift of the edge. The positive peak of the transient (at $4564.3 \mathrm{eV}$ ) lies between the A and B MS features of the iodide spectrum and is therefore related to the changes in the solvent cage around iodine compared to iodide, and so are also the changes observed at higher energies.

2.1.3. $L_{1,3}-E d g e$ Spectra of lodine. With the static iodide spectra and the transient spectra, we can extract the XAS spectra of iodine, but this requires a precise knowledge of the photolysis yield. ${ }^{32}$ In the present case, it cannot be derived from laser-only measurements ${ }^{48}$ because at the concentrations used here (50 and $100 \mathrm{mM}$ ), the CTTS absorption is saturated, while the optical absorption by aqueous $\mathrm{I}^{0}$ (in the $230-300 \mathrm{~nm}$ region ${ }^{38}$ ) is weak and is shaded by the strong absorption of the solvated electron. Therefore, as an alternative way (see details in Supporting Information SIV), we combined the ultrafast optical measurements with the static and the transient $\mathrm{L}_{1}$ and $\mathrm{L}_{3}$ spectra, obtaining values of the fractional population of the photo excited 


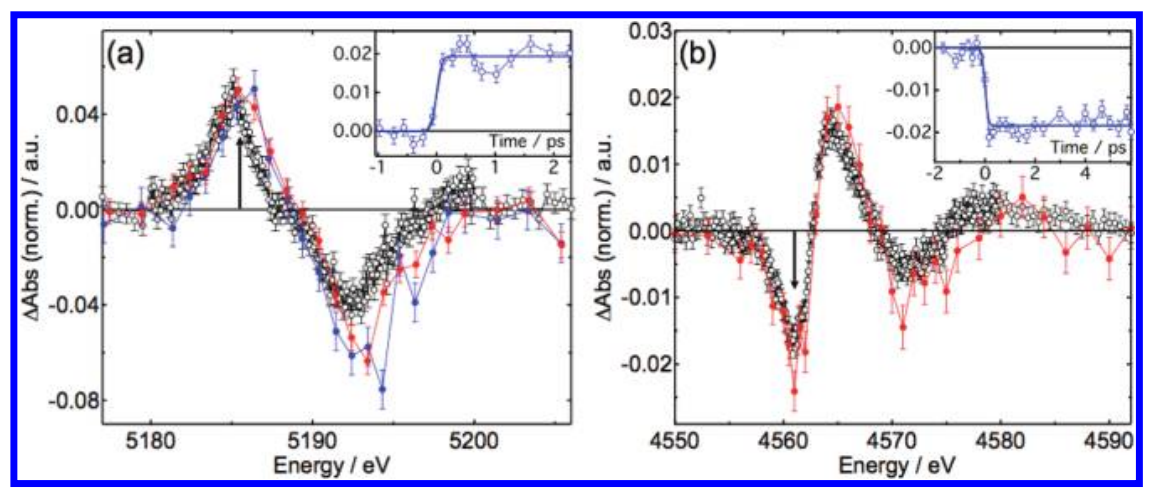

Figure 3. (a) Normalized transient $\mathrm{L}_{1}$ edge spectrum of photoexcited aqueous iodide at $50 \mathrm{ps}$ (black dots), $300 \mathrm{fs}$ (red dots), and $850 \mathrm{fs}$ (blue dots) time delays after excitation; (b) same as panel a but for the transient $\mathrm{L}_{3}$ edge spectrum. The sub-picosecond transients were multiplied by a factor of 1.75 in order to match the value of the $50 \mathrm{ps}$ transient at the maximum of the positive feature. The insets show the time traces recorded with femtosecond resolution at $5185.5 \mathrm{eV}$ (a) and $4561 \mathrm{eV}$ (b), denoted by arrows, together with their fits by a step function with a rise time of $250 \mathrm{fs}$ corresponding to the cross correlation of the optical and X-ray pulses. ${ }^{46}$

species of $4.8 \%$ for the $50 \mathrm{mM}$ sample and $3 \%$ for the $100 \mathrm{mM}$ sample, at 50 ps time delay under our experimental conditions. After normalization to the edge jump (see Supporting Information for details), we retrieve the $\mathrm{I}^{0}$ spectrum by scaling the normalized transient spectrum to the photolysis yield $f(t)$ at a given time delay $t$, and add it to the normalized iodide spectrum according to:

$$
\mu_{\text {norm }}^{I^{0}}=\frac{\Delta A^{\text {norm }}(E, t)}{f(t)}+\mu_{\text {norm }}^{I^{-}}
$$

where $\Delta A^{\text {norm }}$ is the difference absorption normalized to the absorption edge jump of the umpumped sample. The recovered iodine spectra are shown as points in Figures $1 \mathrm{a}$ and $2 \mathrm{a}$ for the $\mathrm{L}_{1}$ and $L_{3}$ edges, respectively. As can be seen from Figure $1 a$, the $L_{1}$ edge shifts by $3.0 \pm 0.6 \mathrm{eV}$ from $\mathrm{I}^{-}$to $\mathrm{I}^{0}$, while Figure 2a shows a shift by $3.2 \pm 0.5 \mathrm{eV}$ at the $\mathrm{L}_{3}$ edge. The agreement between these two shifts suggests that the negative feature in the $L_{3}$ transient results mainly from an edge shift due to the change of oxidation state.

2.2. Femtosecond Transients. Figure 3 a compares the $\mathrm{L}_{1}$ transients recorded at $300 \mathrm{fs}, 850 \mathrm{fs}$, and 50 ps time delay, and Figure $3 \mathrm{~b}$ compares the $\mathrm{L}_{3}$ transients recorded at $300 \mathrm{fs}$ and $50 \mathrm{ps}$ time delay. The sub-picosecond $\mathrm{L}_{1}$ transients exhibit a weak broadening (typically $<1 \mathrm{eV}$ ) with respect to the 50 ps transient, of the high energy side of both their positive and negative features. The broadening of the positive $2 s-5 p$ resonance feature shows similarity to the broadening seen in the transients at much later times (see Figure 1). Whereas in the latter this is clearly due to formation of diiodide species, which is diffusion controlled, in the femtosecond data this is excluded. As will be shown later by QC calculations and by $\mathrm{MD}$ simulations, the broadening can be explained in terms of formation of a shortlived $\mathrm{I}^{0}\left(\mathrm{OH}_{2}\right)$ complex, which has indeed a blue-shifted $2 \mathrm{~s}-5 \mathrm{p}$ resonance.

Contrary to the $\mathrm{L}_{1}$ edge, a broadening or shift is less clear at the $\mathrm{L}_{3}$ edge. This may be due to the fact that, in the latter case, the transient signal results from a combination of a change of oxidation state and changes (in energy and intensity) of the MS features due to the structural rearrangements, making the trends less trivial than in the case of the atomic $2 s-5 p$ resonance. In addition, considering that the MS features are caused by scattering events over the entire solvation shell extending up to several angstroms and comprising several water molecules, the structural contribution of the $\mathrm{I}^{0}\left(\mathrm{OH}_{2}\right)$ complex, with only one nearby water molecule, is expected to be weak.

The insets of Figure 3 show the pump-probe time scans at the $2 s-5 p$ atomic resonance $(5185.5 \mathrm{eV})$ and at the first negative feature in the $\mathrm{L}_{3}$ edge transient $(4561 \mathrm{eV})$. Both exhibit a similar behavior with a sharp rise followed by a constant signal, which is reproduced by the full trace, generated using a step function with a rise time of $\sim 250 \mathrm{fs}$, corresponding to the cross-correlation of the laser and X-ray pulses. ${ }^{46}$

\section{MOLECULAR DYNAMICS SIMULATIONS AND QUANTUM CHEMICAL CALCULATIONS}

The analysis of the above results requires detailed molecular dynamics simulations and quantum chemical calculations, which we present in the following paragraphs, before making contact with the experimental results in the discussion paragraph.

3.1. Solvent Shell Structure around lodine. As already mentioned, the RDFs from the QM/MM MD simulations gave the best agreement of the simulated EXAFS spectrum with the experimental static iodide $\mathrm{L}_{3}$ EXAFS spectrum. ${ }^{42}$ To analyze the changes that we measure at 50 ps time delay (Figures 1 and 2), we need to simulate the RDFs of $\mathrm{I}^{0}$ in an equilibrated water solvent. The $\mathrm{I}^{0}-\mathrm{O} / \mathrm{H}$ RDFs were simulated by both classical and $\mathrm{QM} / \mathrm{MM} \mathrm{MD}$ simulations. The equilibration was carried out over $10 \mathrm{~ns}$ in the Classical MD simulations and over $15 \mathrm{ps}$ in the $\mathrm{QM} / \mathrm{MM}$ MD. ${ }^{42}$ The $\mathrm{I}^{0} \mathrm{RDF}$ are shown in Figure 4 along with the QM/MM MD simulations of the aqueous $\mathrm{I}^{-} \mathrm{RDFs}^{42}$ Both types of MD simulations show an increase of the $\mathrm{I}-\mathrm{O}$ distances from iodide to iodine, but the most dramatic changes show up on the I-H RDF, where the first peak shifts on average by well over $1 \AA$ from iodide to iodine. While for iodide the first peak of the $\mathrm{I}-\mathrm{H}$ RDF lies at smaller distances than that of the I-O RDF, for iodine it is at almost the same distance as the first peak of the $\mathrm{I}-\mathrm{O}$ $\mathrm{RDF}$, but it is broader and has a long tail extending to larger distances. Finally, the I-H RDF shows clear modulations in the case of iodide, but the separation between solvent shells is less clear-cut in the iodine case. All this indicates that the majority of the hydrogen atoms rotate and point away from the iodine atom, contrary to the iodide case. The magnitude of the $\mathrm{I}-\mathrm{O}$ and $\mathrm{I}-\mathrm{H}$ expansions are significantly larger in the classical $\mathrm{MD}(\sim 0.7$ and $1.6 \AA$, respectively) compared to the QM/MM simulations 


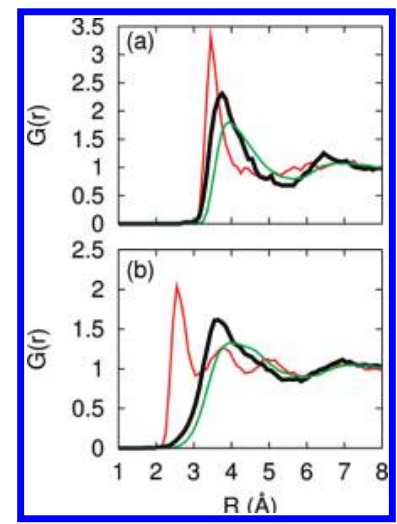

Figure 4. $\mathrm{I}-\mathrm{O}(\mathrm{a})$ and $\mathrm{I}-\mathrm{H}(\mathrm{b})$ radial distribution functions (RDF) of aqueous iodide (red lines, same as in ref 42) and iodine for the classical $\mathrm{MD}$ (green lines) and the QM/MM MD (black lines). The QM/MM MD simulations represent an equilibration over $15 \mathrm{ps}$ with a single $\mathrm{I}^{-} / \mathrm{I}^{0}$ described at DFT level of theory. The $\mathrm{I}^{0}-\mathrm{O} / \mathrm{H}$ RDFs were equilibrated for $10 \mathrm{~ns}$ in the CMD simulations.

( $\sim 0.3 \AA$ and $1.0 \AA$, respectively). The values and trends for the classical MD here are in good agreement with previous studies. ${ }^{9}$

Integration of the iodide RDFs to $5.0 \AA$ (I-O) and $3 \AA(\mathrm{I}-\mathrm{H})$ indicates that the coordination number (i.e., first solvation shell) is $8-9$ oxygen atoms and 7-8 hydrogen atoms, in good agreement with Koneshan et al. ${ }^{9}$ For iodine, integrated to $5.5 \AA$ (I-O) and $6.0 \AA(\mathrm{I}-\mathrm{H})$, the coordination numbers are $20-25$ and $50-60$ for the oxygen and hydrogen atoms, respectively, reflecting the diffuse nature of the solvent shell. As already mentioned, around iodine the majority of the water molecules are oriented so that the oxygen atom lies closer to the iodine than the $\mathrm{H}$ atoms; however, the $\mathrm{I}-\mathrm{H} \mathrm{RDF}$ shows a small probability of some hydrogen atoms being closer to the iodine atom. The integral of this region between $\sim 2.25$ and $3.25 \AA$, that is, the region of $\mathrm{I}-\mathrm{H}$ probability at smaller distances than the main $\mathrm{I}-\mathrm{O}$ peak, amounts to 3-4 hydrogen atoms, which represents a tiny portion of the hydrogen atoms of the first shell around iodine.

Previous instances of significant cage expansions upon electronic excitation of a solute were reported in the case of doped condensed van der Waals (rare gas and hydrogen) crystals, but were due to a repulsive interaction of the dopant excited state with its environment. ${ }^{49,50}$ Indeed in the latter case, the excited state is a diffuse Rydberg orbital, whose overlap with the closed shell structure of the surrounding species is largely dominated by repulsive interactions. Such a scenario cannot be operative here since the wave function of $\mathrm{I}^{0}$ is less diffuse than that of $\mathrm{I}^{-}$. Rather, based on the fact that the $\mathrm{I}-\mathrm{O}$ distance increase is accompanied by a reversal of the direction of most of the $\mathrm{O}-\mathrm{H}$ bonds, we attribute the expansion to the hydrophobic effect, that is, the preference of water molecules to interact with each other more than with the solute, in line with the conclusion of Lynden-Bell, Rasaiah, and co-workers ${ }^{8,9}$ based on classical MD simulations. To verify this hypothesis we present in Supporting Information SII.3, QC geometry optimizations at the CCSD level of $\mathrm{I}^{-}$$\left(\mathrm{H}_{2} \mathrm{O}\right)_{n}$ and $\mathrm{I}^{0}\left(\mathrm{H}_{2} \mathrm{O}\right)_{n}$ clusters with $n=1-4$. While our results for the iodide clusters agree with previous studies, ${ }^{51,52}$ similar calculations are lacking for the neutral species. This is probably because the $\mathrm{I}^{0}\left(\mathrm{OH}_{2}\right)$ potential energy surface is very shallow (see ref 53 and Figure S2), and therefore, finding the minimum energy geometry can be challenging. Figure S3 shows the evolution of the $n=1-4$ clusters for both iodide and iodine, and their detailed

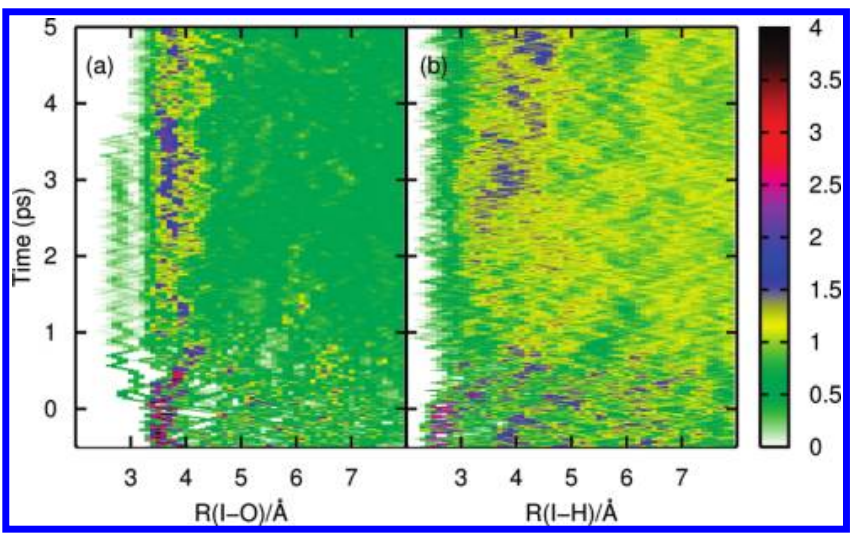

Figure 5. (a) Time evolution of the $\mathrm{I}^{0}-\mathrm{O}$ radial distribution for the first $5 \mathrm{ps}$ following the removal of an electron from iodide. These distributions are obtained from an average over 15 starting configurations over the entire time range; (b) same for $\mathrm{I}^{0}-\mathrm{H}$. Note the departure of both $\mathrm{RDF}$ from the iodide equilibrium configuration before $t=0$.

structural parameters are given in Table S4. Around iodide, a symmetric cluster forms as water molecules are added with a slight gradual increase of the $\mathrm{I}-\mathrm{O}$ and $\mathrm{I}-\mathrm{H}$ distances with $n$, while one proton points toward the ion. The symmetric arrangement of the water molecules around the anion is not surprising considering that the electrostatic interactions between $\mathrm{I}^{-}$and the water dipoles are the dominant intermolecular ones in these structures. The distance increase as a function of $n$ is due to the finite size effect of the cluster as the remaining hydrogen of each molecule tries to form a hydrogen bond network with the other molecules. The distance of the hydrogen bonds gradually increases from $1.88 \AA(n=2)$ to $2.19 \AA(n=4)$. However, due to the small size of the cluster, this is still significantly smaller than the $\sim 3 \AA$ distance of hydrogen bonds typically observed in bulk water. ${ }^{54}$

For $\mathrm{I}^{0}$ starting with $n=1$, the $\mathrm{I}-\mathrm{O}$ distance is shorter than for iodide, while the opposite occurs for the $\mathrm{I}-\mathrm{H}$ distance, showing that the hydrogen atoms point away from the iodine atom, as expected for the fact that the more electron rich part of the water molecule (its oxygen end) will now be attracted by the high electron affinity iodine atom. However, the $\mathrm{I}-\mathrm{OH}_{2}$ interaction potential is very shallow with a well depth of $0.05 \mathrm{eV}$ according to our quantum chemical calculations (Figure S2). Adding water molecules does not lead to a direct interaction with iodine, but rather the additional water molecules form hydrogen bonds with each other. This suggests the initial formation of small hydrogen bond network between water molecules, while one $\mathrm{H}_{2} \mathrm{O}$ molecule remains close to $\mathrm{I}^{0}$. It is likely that adding more water molecules will end up in the formation of a complete hydration shell of H-bonded molecules, but computational expense and the very flat nature of the iodine potential energy surface make it unrealistic to perform these optimizations on larger structures.

3.2. Solvent Cage Rearrangement at Early Times. We tested the hypothesis of the formation of a full hydration shell (cavity formation) by means of QM/MM molecular dynamics (see Supporting Information SII.2 for details). The results thus obtained also provide the basis for the interpretation of the subpicosecond XAS transients.

The simulations were carried out with a quantum part consisting of $\mathrm{I}^{0}$ and 9 water molecules (corresponding to the first complete solvation shell of the iodide) within a bath of 1183 classical water molecules. Figure 5 shows the time evolution of 


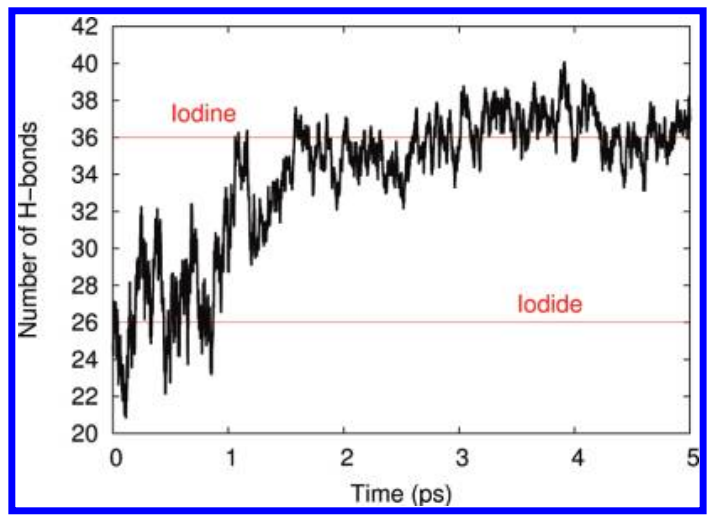

Figure 6. Time evolution of the number of hydrogen bonds within a radius of $7 \AA$ around the iodine atom. This is obtained from an average over 15 starting configurations. The red lines show the number of $\mathrm{H}$-bonds at equilibrium for iodide and iodine.

the $\mathrm{I}^{0}-\mathrm{O}(\mathrm{a})$ and $\mathrm{I}^{0}-\mathrm{H}(\mathrm{b}) \mathrm{RDFs}$ over the first 5 ps after removal of the electron from iodide. These results are an average of 15 starting configurations for each time step. Within $200-300 \mathrm{fs}$, there is break-up of the first shell with most molecules moving away from iodine while approximately one moves closer (Figure 5a), and forms a complex with the iodine atom. This complex formation may be favored by the anisotropy of the solvent cage around the iodide, which has previously been pointed out. ${ }^{42,44}$

There is also a prompt expansion of the $\mathrm{I}-\mathrm{H}$ distance within about 200 fs. Figure S4 shows the integral of the I-O RDF (Figure 5) between 0 and $3.2 \AA$, as a function of time. It indicates that, beyond $\sim 500 \mathrm{fs}$, the probability of finding a water molecule at a distance of $\sim 2.8 \AA$ of the iodine atom is $\sim 0.4$. This distance is in good agreement with that found for the $\mathrm{I}^{\mathrm{0}}\left(\mathrm{H}_{2} \mathrm{O}\right)_{4}$ cluster (Figure S3 and Table S4). Up to $\sim 2.0 \mathrm{ps,} \mathrm{the} \mathrm{solvation} \mathrm{shell}$ remains very diffuse and unstructured as can be seen on both $\mathrm{I}-\mathrm{O}$ and $\mathrm{I}-\mathrm{H} \mathrm{RDFs}$. From about 2 ps onward, one observes that the first shell starts to form and to become gradually structured. This is visible both in the increase of the number of oxygen atoms but also in that of hydrogen atoms that have on average their probability at larger distances than the $\mathrm{O}$ atoms; that is, they point toward the bulk rather than toward the solute. This process continues up to about $3.5 \mathrm{ps}$, as witnessed by the value of the I-O RDF that approaches 2.5 at $\sim 3.8 \AA$ as in Figure 4a. Likewise, for the I-H RDF, during this time span, the value of $1.5-2.0$ is reached as in Figure $4 \mathrm{~b}$.

At $\sim 3.5 \mathrm{ps}$, the water molecule which formed a complex with the iodine species merges with the bulk of the solvent. This occurs as the new solvation shell is completing formation of a structured hydrogen bond network, as seen in Figure 6, where the number of hydrogen bonds within a sphere of $7 \AA$ around the iodine is plotted as a function of time following the removal of an electron from iodide. A hydrogen bond is defined whenever two neighboring water molecules are at an $\mathrm{O}-\mathrm{O}$ distance of $\leq 3 \AA$ and an $\mathrm{HOH} \cdots \mathrm{O}$ angle of $\leq 20^{\circ} .55$ One can see that there is an increase of approximately 10 hydrogen bonds in this region, from $\sim 26$ in the iodide case to $\sim 36$ in the iodine case, over the time scale of approximately 3 ps and it clearly indicates the formation of a solvent cavity around the solute, which overwhelms the interaction the lone water molecule has with the iodine atom. This preference of the water molecules to interact with each other rather than with the solute is the hallmark of hydrophobicity.

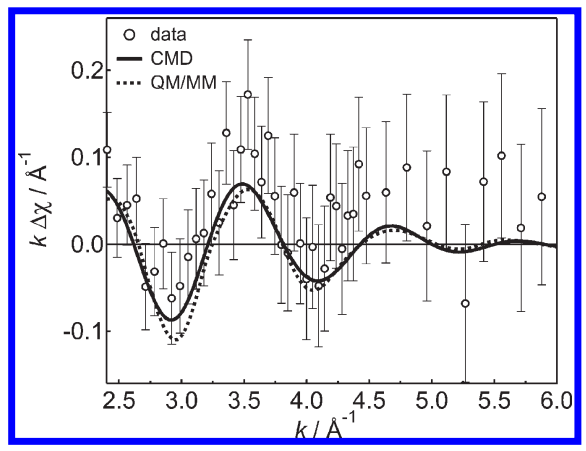

Figure 7. Experimental transient spectrum $\mathrm{L}_{3}$ EXAFS spectrum in $k$ space binned by a factor of 3 in order to increase the statistics (circles with error bars) and simulated transient spectra (using the procedure described in Supporting Information SIII) for the case of the classical $\mathrm{MD}$ (full line) and $\mathrm{QM} / \mathrm{MM} \mathrm{MD}$ (dotted line). The Classical MD transient was shifted by $3 \mathrm{eV}$ and the $\mathrm{QM} / \mathrm{MM}$ one by $0.5 \mathrm{eV}$ in energy space in order to best match the experimental data. For the simulated transients, we used for iodide the EXAFS spectrum obtained from the $\mathrm{QM} / \mathrm{MM}$ simulations. ${ }^{42}$

It also agrees with the definition by Linden-Bell and Rasaiah of hydrophobic hydration around zero charge solutes. ${ }^{8,9}$

\section{DISCUSSION}

We now use the results of the previous section to discuss the picosecond and femtosecond X-ray absorption spectra of Figures 1 and 2.

4.1. Picosecond XAS Studies. Considering that, at $50 \mathrm{ps}$ time delay, the sample contains only iodine as photoproduct (see Supporting Information SIV), we can use the picosecond transients (Figures 1 and 2) to extract the solvent shell structure around iodine. Because of the weak above-edge modulations at the $\mathrm{L}_{1}$ edge, ${ }^{35}$ its transient is not useful for a structural analysis. Rather, we need to analyze the XANES and EXAFS features of the retrieved $\mathrm{I}^{0} \mathrm{~L}_{3}$ spectrum of Figure 2a. However, the latter is too noisy for a reliable analysis, in particular in the EXAFS region. ${ }^{56}$ We therefore adopted the approach of ref 57 which consists in directly comparing the transient (difference) experimental spectrum in $k$-space at 50 ps with that generated from the RDFs obtained by MD simulations. The approach based on using the difference spectrum rather than the retrieved spectrum of the photoproduct has the advantage of reducing the number of required parameters by cancellation in the differences. ${ }^{57}$ The experimental transient spectrum converted to $k$-space and scaled by $k$ (see Supporting Information SIII) over the energy range from 4580 to $4700 \mathrm{eV}$ is shown in Figure 7. Note that the experimental data points are on average above the zero difference level. The origin of this vertical offset is unclear, but it is most likely due to a change of atomic background in going from iodide to iodine. Indeed, it is known that the atomic X-ray absorption fine structure (AXAFS) is due to scattering of the photoelectron from the periphery of the absorbing atom. ${ }^{58-60}$ Since the scattering process is determined by the binding energies of the electrons in the various orbitals, and since these change in going from iodide to iodine, it is not unexpected that the background also changes. ${ }^{60}$ At this stage we did not try to account for such effects and we will use the difference spectrum in $k$-space as obtained.

For simulating the transient EXAFS spectrum, we use the fractional population of $\mathrm{I}^{0}$ species at $50 \mathrm{ps}(3 \%$, derived in 


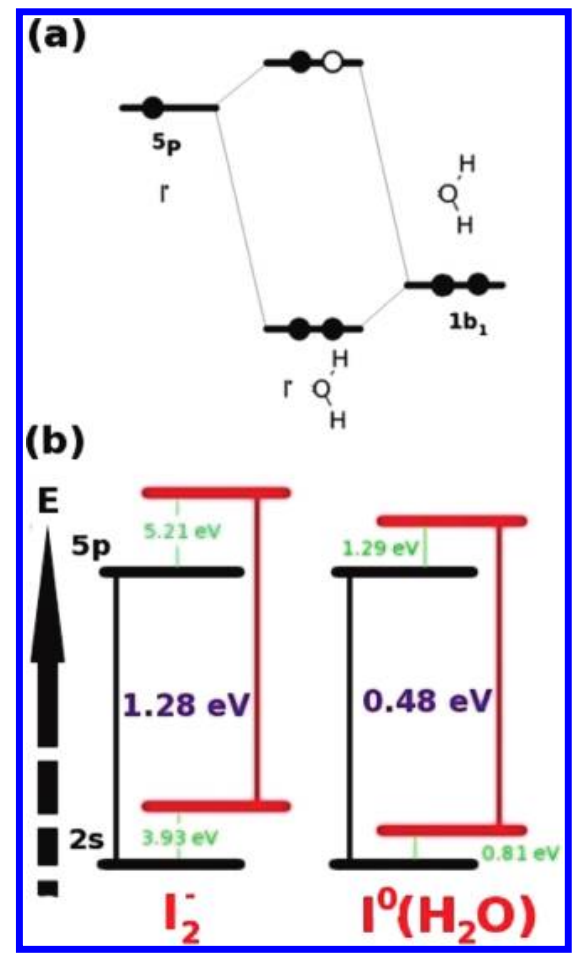

Figure 8. (a) Schematic representation of the orbital diagrams for the $\mathrm{I}^{0}-\mathrm{OH}_{2}$ complex forming a three-electron bond between the $5 \mathrm{p}$ electron of $\mathrm{I}^{0}$ and the $1 \mathrm{~b}_{1}$ orbital of water; (b) calculated energy shifts with respect to the bare iodine atom of the $2 \mathrm{~s}$ and the $5 \mathrm{p}$ orbitals (in green color) upon formation of diiodide and of the $\mathrm{I}^{0}-\mathrm{OH}_{2}$ complex obtained at CCSD level of theory with the DGDZVP basis set. The energy values in blue represent the calculated shifts of the $2 \mathrm{~s}-5 \mathrm{p}$ transition.

Supporting Information SIV.3) and the I-O RDFs of $\mathrm{I}^{-}$and $\mathrm{I}^{0}$ (Figure 4) as input parameters. We neglect the contribution of hydrogen atoms at this point, given the large noise in the transient. The RDFs are used to simulate the EXAFS spectra as described in ref 42 and in Supporting Information SIII. The transient (difference) spectrum is then calculated from the RDFs using eq S2. The results are also shown in Figure 7 for both the classical and QM/MM MD simulations of aqueous $\mathrm{I}^{0}$. The energy shift is used as a fit parameter. The simulated transients for both types of MD simulations agree fairly well with the experimental transient, within the large error bars of the latter and bearing in mind the vertical offset. The main result here is that the experimental data confirms the expansion of the solvent cage from $\mathrm{I}^{-}$to $\mathrm{I}^{0}$, with an increase of the cage radius (measured on the I-O distance) of 5-20\% (depending on the type of simulations).

4.2. Sub-Picosecond XAS Studies. The time scans shown in the insets of Figure 3 are characterized by a step function with a rise determined by the cross correlation of our experiment ( $\sim 250 \mathrm{fs})$. This implies a prompt formation (as expected) of the $\mathrm{I}^{0}$ species. The lack of evolution of the signal at later times, which would have been anticipated based on Figure 5, is probably due to the large noise and to the weak effects that are seen at early times in Figure 3, namely, the broadening on the blue side. However, the formation of an $\mathrm{I}^{0}\left(\mathrm{OH}_{2}\right)$ complex within the solvent cage (Figure S3 and Figure 5) explains the broadening of the $2 s-5 p$ resonance in the sub-picosecond transients of Figure 3a. Indeed, by analogy with $\mathrm{I}_{2}{ }^{-}$, combining $\mathrm{I}^{-}\left(5 \mathrm{p}^{6}\right)$ and
$\mathrm{I}^{0}\left(5 \mathrm{p}^{5}\right)$ to form $\mathrm{I}_{2}{ }^{-}$populates the lower bonding $\mathrm{p} \sigma$ orbitals with two electrons, whereas the higher energy anti-bonding $\mathrm{p} \sigma^{*}$ orbital contains only one electron, making it the sole orbital accessible from the $2 \mathrm{~s}$ core orbital. This leads to a blue-shifted resonance, which explains the broadening (in Figure 1c) of the $2 s-5 p$ resonance on its high energy side, as at 2 ns the sample contains $\mathrm{I}^{0}, \mathrm{I}_{2}{ }^{-}$, and even some $\mathrm{I}_{3}{ }^{-}$(Figure S6). Likewise, combining $\mathrm{I}^{0}\left(5 \mathrm{p}^{5}\right)$ with the $1 \mathrm{~b}_{1}$ orbital of water leads to the formation of a similar 3-electron bond, with the upper antibonding orbital being the only one accessible from the 2 s orbital as shown in Figure 8a, and this behavior seems general to all halogen atoms. Indeed, formation of a stable $\mathrm{Cl}^{0}\left(\mathrm{OH}_{2}\right)$ complex was previously reported in electron spin resonance studies, which were supported by quantum-chemical calculations, pointing to a three-electron $\left(\sigma^{2} \sigma^{1 *}\right)$ bonding. ${ }^{61}$ The presence of a $\mathrm{Br}^{0}\left(\mathrm{OH}_{2}\right)$ complex was also found in Monte Carlo simulations of $\mathrm{Br}^{0}$ in water. $^{36}$ In the case of $\mathrm{I}^{0}$, the complex is weakly bound (Figure $\mathrm{S} 2$ ), with a typical well depth of $\sim 50 \mathrm{meV}$. Because of their larger electron affinities, $\mathrm{Cl}$ and $\mathrm{Br}$ are expected to form more stable complexes with water than $\mathrm{I}^{0}$, with the $\mathrm{Br}$ complex being less bound than the $\mathrm{Cl}$ one. ${ }^{62}$

To be more quantitative, we calculated the energy shift of the $2 s-5 p$ resonance (see Supporting Information SII.4) with respect to the bare iodine atom for $\mathrm{I}_{2}{ }^{-}$and $\mathrm{I}^{0}\left(\mathrm{OH}_{2}\right)$ and the results are shown in Figure $8 \mathrm{~b}$. For both species, the $2 \mathrm{~s}$ and the $5 \mathrm{p}$ orbitals shift to higher energies, but the magnitude is larger for the latter, so that the overall shift of the $2 s-5 p$ resonance amounts to $\sim 1.3 \mathrm{eV}$ for diiodide and $\sim 0.5 \mathrm{eV}$ for $\mathrm{I}^{0}\left(\mathrm{OH}_{2}\right)$ in good agreement with the experimental observations $(\sim 1 \mathrm{eV}$ in Figure $1 \mathrm{c}$ and $\sim 0.7 \mathrm{eV}$ in Figure $3 \mathrm{a}$ ).

Since the average probability of finding the lone water molecule close to the iodine atom during the first $3 \mathrm{ps}$ is around 0.4 (Figure S4), this implies that the sub-picosecond transients (Figure 3) contain contributions from both the bare and the complexed iodine. Given that the latter is slightly blue-shifted with respect to the former, this explains the broadening of the $2 s-5 p$ resonance on its blue side, rather than a blue shift of the entire band. The fact that the $\mathrm{L}_{1}$-edge transients at 300 and $850 \mathrm{fs}$ agree with each other but deviate from the 50 ps one (Figure 1a) is also in line with the $\sim 3.5$ ps lifetime of the complex (Figure 5 and Figure S4).

On the basis of Figure 6, the lifetime of the complex seems to be correlated to the time it takes to complete a network of $\mathrm{H}$-bonded molecules around the solute. Only once the cavity is fully formed can the water molecule of the complex be attracted by the bulk. This is probably due to the fact that the water molecule of the complex has its hydrogens pointing toward the cavity boundary, which has an excess of oxygen atoms. This last step completes the formation of a hydrophobic cavity around the neutral iodine. A computer animation made from the MD simulations is shown in the Supporting Information, illustrating the process of cage expansion, complex formation, and detachment of the water molecule from the complex.

It is interesting to cast these results in the context of the $\mathrm{I}^{-}\left(\mathrm{H}_{2} \mathrm{O}\right)_{n=3-10}$ cluster studies by Neumark and co-workers using photoelectron imaging. ${ }^{63}$ They measured the vertical detachment energies as a function of time after exciting the CTTS bands of the cluster, and found a drop of $\sim 50 \mathrm{meV}$ after a few tens of picoseconds, which they attribute to the loss of the iodine atom. This value is remarkably close to the binding energy of the $\mathrm{I}\left(\mathrm{OH}_{2}\right)$ complex (Figure S2). In the present case, the separation of the water molecule from the complex it formed 
with the $\mathrm{I}^{0}$ atom would be the analogue of the loss of the $\mathrm{I}^{0}$ atom in the cluster. However, the processes are somewhat different. Indeed, and as seen in Figure S3 and is known in the literature, ${ }^{64}$ $\mathrm{I}^{-}$sits at the surface of the cluster and a full solvation is probably never achieved (even with 10 molecules). The ensuing dynamics around the $\mathrm{I}^{0}$ atom are most likely determined by these initial conditions and the detachment of $\mathrm{I}^{0}$ is then governed by thermal effects, which require long time scales (tens of picoseconds ${ }^{63}$ ) to affect the cluster. In the present case, the time scale of complex dissociation is much shorter and is probably governed by the attractive forces from the solvation shell, once it has completed its rearrangement.

In their studies of electron production under resonant single photon excitation of the lowest CTTS state of aqueous iodide, Bradforth and co-workers ${ }^{39,65}$ and Laubereau and co-workers ${ }^{37}$ interpreted their results using the theoretical model of Staib and Borgis on the related system of aqueous chloride. That is, following excitation, a halogen/electron pair forms, which may undergo geminate recombination or else dissociate to produce a hydrated electron. ${ }^{66,67}$ The experimental results show that, in the case of aqueous iodide, short-range ejection of the electron to the $\mathrm{I} / \mathrm{e}^{-}$pair occurs within $200 \mathrm{fs}$, followed by stabilization of the contact pair via solvent rearrangement, complete within $\sim 2$ ps. Geminate recombination takes place with a lifetime on the order of 30 ps, with $\sim 25 \%$ of electrons undergoing diffusive escape with an upper limit lifetime of $\sim 70$ ps. ${ }^{68}$ These results were inferred from the kinetics of electron production but the contact pair has never been identified spectroscopically. Even recent UV photoelectron studies of CTTS photoexcited iodide in liquid micro jets ${ }^{69,70}$ failed to provide spectroscopic evidence of the contact pair. Although it may also be invoked to explain the present results, it is difficult to reconcile with the fact that, as already mentioned, the predominantly 3-photon process in our case makes it unlikely that a contact pair occurs. In addition, it is also difficult to reconcile with the fact that should it be present, it is unlikely to yield a blue shift of the $2 s-5$ p resonance, but would either be at the energy or to the red of the latter for the bare atom.

In summary, the picture that emerges from the above results is that, upon abstraction of the electron from iodide, there is a prompt expansion of the cage within 200-300 fs, but the development of hydrophobic solvation is a complex process which requires a time span of 3-4 ps. During this time, a weakly bonded transient $\mathrm{I}^{0}\left(\mathrm{OH}_{2}\right)$ complex is formed with a probability of $\sim 40 \%$, which survives the time it takes to complete the formation of a new shell of water molecules around iodine. This new shell is characterized by an expansion of the $\mathrm{I}-\mathrm{O}$ distances, but more important by a reorientation of most of the $\mathrm{H}$ atoms away from the solute and toward the bulk solvent, such as to allow them to build a network of $\mathrm{H}$-bonded water molecules. In bulk water, this implies reorientational dynamics of the molecules, ${ }^{27,71}$ which requires typically $2-3$ ps. $^{72,73}$ The recent simulations by Laage et al. ${ }^{27}$ show that the rate of water reorientation in the vicinity of the hydrophobic solutes is decreased only moderately (by merely a factor of 2 ), which would be on the same time scale as what we determine in the $\mathrm{QM} / \mathrm{MM} \mathrm{MD}$ simulations. Once the $\mathrm{H}$-bonds have reached their steady state regime is the lone water molecule pulled toward the bulk of the solvent, probably because the interaction with the water molecules of the cavity overwhelms the $\mathrm{I}^{0}-\mathrm{OH}_{2}$ interaction.

The present experimental results, consisting of picosecond and femtosecond X-ray absorption spectra, were interpreted with the help of quantum chemical calculations and molecular dynamics simulations, allowing us to unravel the entire evolution of the system from the hydrophilic hydration in the case of iodide to the hydrophobic one in the case of iodine. With the advent of the free electron lasers, which provide very intense ultrashort pulses of X-ray, it is now possible to carry out detailed X-ray absorption studies of the present system with femtosecond resolution and a greatly improved signal-to-noise ratio, such that the details revealed by the $\mathrm{MD}$ simulations can fully be verified.

\section{ASSOCIATED CONTENT}

S Supporting Information. Description of the static, picosecond and femtosecond X-ray absorption experiments, power dependence of the solvated electron signal, description of molecular dynamics simulations, quantum-chemical calculations and simulations of the transient EXAFS spectrum, extraction of the photolysis yield, figure of the $\mathrm{I}^{-} / \mathrm{I}^{0}\left(\mathrm{H}_{2} \mathrm{O}\right)_{n=1-4}$ clusters and table of their structural parameters; computer animation of the first 5 ps of the dynamics ( $\mathrm{mpg}$ ). This material is available free of charge via the Internet at http://pubs.acs.org.

\section{AUTHOR INFORMATION}

\section{Corresponding Author}

majed.chergui@epfl.ch

\section{Present Addresses}

${ }^{\ddagger}$ Pacific Northwest National Laboratory, 902 Battelle Blvd, Richland, WA 99352, USA

${ }^{\perp}$ Physical Biology Center for Ultrafast Science \& Technology, California Institute of Technology, Pasadena, CA 91125, USA

"European XFEL Facility, Albert-Einstein Ring 19, D-22761 Hamburg, Germany

\section{ACKNOWLEDGMENT}

This work was supported by the Swiss NSF for support via contracts 200021-107956, 200021-105239/1 and 200020116533, the Sekretariat für Bildung und Forschung via contract COST D35 060016 and the NCCR-MUST. We thank Prof. Ursula Röthlisberger for computer time and for allowing us to use the as yet unpublished Dispersion Corrected Atom-Centred Potentials for iodine.

\section{REFERENCES}

(1) Pratt, L. R. Annu. Rev. Phys. Chem. 2002, 53, 409.

(2) Dill, K. A.; Truskett, T. M.; Vlachy, V.; Hribar-Lee, B. Annu. Rev. Biophvs. Biomol Struct. 2005, 34, 173.

(3) Galli, G. Proc. Natl. Acad. Sci. U.S.A. 2007, 104, 2557.

(4) Berne, B. J.; Weeks, J. D.; Zhou, R. H. Annu. Rev. Phys. Chem. 2009, 60, 85 .

(5) Stillinger, F. H. L. Solution Chem. 1973, 2, 141.

(6) Pratt, L. R.; Chandler, D. I. Chem. Phvs. 1977, 67, 3683.

(7) Miller, T. F.; Vanden-Eijnden, E.; Chandler, D. Proc. Natl. Acad. Sci. U.S.A. 2007, 104, 14559.

(8) LyndenBell, R. M.; Rasaiah, J. C. I. Chem. Phvs. 1997, 107, 1981.

(9) Koneshan, S.; Rasaiah, J. C.; Lynden-Bell, R. M.; Lee, S. H. I. Phvs. Chem. B 1998, 102, 4193.

(10) Li, J. L.; Car, R.; Tang, C.; Wingreen, N. S. Proc. Natl. Acad. Sci. U.S.A. 2007, 104, 2626.

(11) Mezger, M.; Reichert, H.; Schoder, S.; Okasinski, J.; Schroder, H.; Dosch, H.; Palms, D.; Ralston, J.; Honkimaki, V.P Natl Acad Sci USA 2006, 103, 18401. 
(12) Mezger, M.; Sedlmeier, F.; Horinek, D.; Reichert, H.; Pontoni, D.; Dosch, H. L.Am. Chem. Soc. 2010, 132, 6735.

(13) Neilson, G. W.; Mason, P. E.; Ramos, S.; Sullivan, D. Philos. Trans. R. Soc., A 2001, 359, 1575.

(14) Sullivan, D. M.; Neilson, G. W.; Fischer, H. E. J. Chem. Phys. 2001, 115, 339.

(15) Guillot, B.; Guissani, Y. L. Chem. Phvs. 1993, 99, 8075.

(16) Filipponi, A.; Bowron, D. T.; Lobban, C.; Finney, J. L. Phys. Rev. Lett. 1997, 79, 1293.

(17) Bowron, D. T.; Filipponi, A.; Lobban, C.; Finney, J. L. Chem. Phys. Lett. 1998, 293, 33.

(18) Bowron, D. T.; Filipponi, A.; Roberts, M. A.; Finney, J. L. Phys. Rev. Lett. 1998, 81, 4164.

(19) Rezus, Y. L. A.; Bakker, H. I. Phys. Rev. Lett. 2007, 99, 148301.

(20) Frank, H. S.; Evans, M. W. I. Chem. Phys. 1945, 13, 507.

(21) Haselmeier, R.; Holz, M.; Marbach, W.; Weingartner, H. J. Phys. Chem. 1995, 99, 2243.

(22) Ishihara, Y.; Okouchi, S.; Uedaira, H. J. Chem. Soc., Faraday Trans. 1997, 93, 3337.

(23) Kaatze, U.; Gerke, H.; Pottel, R. L.Phvs. Chem. 1986, 90, 5464.

(24) Wachter, W.; Buchner, R.; Hefter, G. J. Phys. Chem. B 2006, 110,5147

(25) Rossky, P. J.; Karplus, M. I. Am. Chem. Soc. 1979, 101, 1913.

(26) Zichi, D. A.; Rossky, P. J. L. Chem. Phvs. 1986, 84, 2814.

(27) Laage, D.; Stirnemann, G.; Hynes, J. T. J. Phys. Chem. B 2009, $113,2428$.

(28) Lee, S. H.; Rasaiah, J. C. I. Chem. Phvs. 1994, 101, 6964.

(29) Lee, S. H.; Rasaiah, J. C. I. Phvs. Chem. 1996, 100, 1420.

(30) Bressler, C.; Saes, M.; Chergui, M.; Grolimund, D.; Abela, R.; Pattison, P. I. Chem. Phvs. 2002, 116, 2955.

(31) Bressler, C.; Chergui, M. Chem. Rev. 2004, 104, 1781.

(32) Bressler, C.; Abela, R.; Chergui, M. Z. Kristallogr. 2008, 223, 307.

(33) Bressler, C.; Chergui, M. Annu. Rev. Phvs. Chem. 2010, 61, 263.

(34) Chergui, M. Acta Crystallogr., Sect. A 2010, 66, 229.

(35) Pham, V. T.; Gawelda, W.; Zaushitsyn, Y.; Kaiser, M.; Grolimund, D.; Johnson, S. L.; Abela, R.; Bressler, C.; Chergui, M. J. Am. Chem. Soc. 2007, 129, 1530.

(36) Elles, C. G.; Shkrob, I. A.; Crowell, R. A.; Arms, D. A.; Landahl, E. C. J. Chem. Phys. 2008, 128.

(37) Iglev, H.; Trifonov, A.; Thaller, A.; Buchvarov, I.; Fiebig, T.; Laubereau, A. Chem. Phvs. Lett. 2005, 403, 198.

(38) Moskun, A. C.; Bradforth, S. E.; Thogersen, J.; Keiding, S.J Phys Chem A 2006, 110, 10947.

(39) Chen, X. Y.; Bradforth, S. E. Annu. Rev. Phys. Chem. 2008, 59, 203.

(40) Long, F. H.; Lu, H.; Eisenthal, K. B. J. Chem. Phys. 1989, 91, 4413.

(41) Long, F. H.; Shi, X. L.; Lu, H.; Eisenthal, K. B. J. Phys. Chem. 1994, 98, 7252.

(42) Pham, V. T.; Tavernelli, I.; Milne, C. J.; van der Veen, R. M.; D’Angelo, P.; Bressler, C.; Chergui, M. Chem. Phvs. 2010, 371, 24.

(43) Laio, A.; VandeVondele, J.; Rothlisberger, U. J. Chem. Phys. 2002, 116, 6941.

(44) Wick, C. A.; Xantheas, S. S. I. Phys. Chem. B 2009, 113, 4141.

(45) Saes, M.; Bressler, C.; van Mourik, F.; Gawelda, W.; Kaiser, M.; Chergui, M.; Bressler, C.; Grolimund, D.; Abela, R.; Glover, T. E.; Heimann, P. A.; Schoenlein, R. W.; Johnson, S. L.; Lindenberg, A. M.; Falcone, R. W. Rev. Sci. Instrum. 2004, 75, 24.

(46) Bressler, C.; Milne, C.; Pham, V. T.; ElNahhas, A.; van der Veen, R. M.; Gawelda, W.; Johnson, S.; Beaud, P.; Grolimund, D.; Kaiser, M.; Borca, C. N.; Ingold, G.; Abela, R.; Chergui, M. Science 2009, 323, 489.

(47) Tanida, H.; Kato, K.; Watanabe, I. Bull. Chem. Soc. Jpn. 2003, 76, 1735.

(48) Gawelda, W.; Pham, V. T.; Benfatto, M.; Zaushitsyn, Y.; Kaiser, M.; Grolimund, D.; Johnson, S. L.; Abela, R.; Hauser, A.; Bressler, C.; Chergui, M. Phvs. Rev. Lett. 2007, 98, 057401.
(49) Jeannin, C.; Porrella-Oberli, M. T.; Jimenez, S.; Vigliotti, F.; Lang, B.; Chergui, M. Chem. Phys. Lett. 2000, 316, 51.

(50) Bonacina, L.; Larregaray, P.; van Mourik, F.; Chergui, M. Phys. Rev. Lett. 2005, 95, 015301.

(51) Kim, J.; Lee, H. M.; Suh, S. B.; Majumdar, D.; Kim, K. S. J. Chem. Phys. 2000, 113, 5259.

(52) Majumdar, D.; Kim, J.; Kim, K. S. I. Chem. Phvs. 2000, 112, 101.

(53) Kowal, M.; Gora, R. W.; Roszak, S.; Leszczynski, J. J. Chem. Phys. 2001, 115, 9260.

(54) Laage, D.; Hynes, J. T. Science 2006, 311, 832.

(55) Humphrey, W.; Dalke, A.; Schulten, K. J. Mol. Graphics 1996, $14,33$.

(56) Rehr, J. J.; Albers, R. C. Rev. Mod. Phvs. 2000, 72, 621.

(57) Gawelda, W.; Pham, V. T.; van der Veen, R. M.; Grolimund, D.; Abela, R; Chergui, M.; Bressler, C. I. Chem. Phys. 2009, 130, 124520.

(58) Holland, B. W.; Pendry, J. B.; Pettifer, R. F.; Bordas, J. J. Phys. C: Solid State Phvs. 1978, 11, 633.

(59) Rehr, J. J.; Booth, C. H.; Bridges, F.; Zabinsky, S. I. Phys. Rev. B 1994, 49, 12347.

(60) Ramaker, D. E.; Mojet, B. L.; Koningsberger, D. C.; O'Grady, W. E. L. Phvs.: Condens. Matter 1998, 10, 8753.

(61) Sevilla, M. D.; Summerfield, S.; Eliezer, I.; Rak, J.; Symons, M. C. R. I. Phys. Chem. A 1997, 101, 2910.

(62) Roeselova, M.; Kaidor, U.; Jungwirth, P. J. Phys. Chem. A 2000, 104, 6523.

(63) Kammrath, A.; Verlet, J. R. R.; Bragg, A. E.; Griffin, G. B.; Neumark, D. M. L. Phys. Chem. A 2005, 109, 11475.

(64) Bradforth, S. E.; Jungwirth, P. I. Phvs. Chem. A 2002, 106, 1286.

(65) Kloepfer, J. A.; Vilchiz, V. H.; Lenchenkov, V. A.; Chen, X. Y.;

Bradforth, S. E. I. Chem. Phvs. 2002, 117, 766.

(66) Staib, A.; Borgis, D. L. Chem. Phvs. 1996, 104, 9027.

(67) Borgis, D.; Staib, A. L.Phvs.: Condens. Matter 1996, 8, 9389.

(68) Kloepfer, J. A.; Vilchiz, V. H.; Lenchenkov, V. A.; Germaine,

A. C.; Bradforth, S. E. I. Chem. Phvs. 2000, 113, 6288.

(69) Lubcke, A.; Buchner, F.; Heine, N.; Hertel, I. V.; Schultz, T. Phvs. Chem. Chem. Phvs. 2010, 12, 14629.

(70) Tang, Y.; Shen, H.; Sekiguchi, K.; Kurahashi, N.; Mizuno, T.; Suzuki, Y. I.; Suzuki, T. Phvs. Chem. Chem. Phvs. 2010, 12, 3653.

(71) Pal, S. K.; Peon, J.; Bagchi, B.; Zewail, A. H. J. Phys. Chem. B 2002, 106, 12376.

(72) Laage, D.; Hynes, J. T. Chem. Phvs. Lett. 2006, 433, 80.

(73) Laage, D.; Hynes, J. T. Proc. Natl. Acad. Sci. U.S.A. 2007, 104, 11167. 